\title{
Sonar Based Map Building for a Mobile Robot
}

\author{
Kok Seng CHONG \\ kok.seng.chong@eng.monash.edu.au
}

\author{
Lindsay KLEEMAN \\ lindsay.kleeman@eng.monash.edu.au
}

\author{
Intelligent Robotics Research Centre (IRRC) \\ Department of Electrical and Computer Systems Engineering \\ Monash University, Victoria 3168, Australia \\ http://calvin.eng.monash.edu.au/IRRC/
}

\begin{abstract}
This paper describes a mobile robot equipped with a sonar sensor array, Werrimbi, in a guided feature based map building task in an indoor environment. Common indoor landmarks such as planes, corners and edges are located and classified with a multiple transducer sensor array. Accurate odometry information is derived from a pair of narrow unloaded encoder wheels. Discrete sonar observations are incrementally merged into partial planes to produce a realistic representation of environment. Collinearity constraints among features are exploited to enhance state estimation. The map update utilises Julier-Uhlmann Kalman Filter (JUKF) which improves the accuracy of covariance propagation through nonlinear equations and eliminates the need to derive Jacobian matrices. Correlation among map features and robot location are explicitly represented. Partial planes are also used to eliminate phantom targets caused by sonar specular reflection.
\end{abstract}

\section{Introduction}

The aim of this work is to implement an autonomous mobile robot capable of navigating in an a priori unknown indoor environment using a sonar sensor array. Such systems require map building capability, which is a cyclic process of moving to a new position, sensing the environment and incrementally registering findings into a map to assist subsequent motion planning. Map integrity cannot be sustained by odometry alone due to errors introduced by wheel distortion and slippage. Extereoceptive sensing provides supplementary information to odometry.

Sonar systems are often associated with grid based strategies because of the wide beamwidth of ultrasonic transducers [13]. Grid map update schemes range from Bayesian [10], Evidential [17] to Fuzzy [16]. Localisation with a grid map can be complex. A localisation scheme for a laser system has been developed in [5], whereas [3, 15] do not incorporate localisation. Feature based mapping schemes have become more commonplace $[4,18]$ after Kuc and Siegel [8] presented a method for discriminating planes, corners and edges using sonar data gathered at two positions. Later Kleeman and Kuc [7] developed a sonar sensor which allows target classification at one position. However, all sonar observations are discrete elements.
Therefore, while navigating along a wall, the robot sees the wall not as an entity but as a set of approximately collinear planar elements. Data fusion methods associated with feature based mapping include Kalman Filter [1,12,19], maximum likelihood estimation [11] and heuristic rules [3]. Another important consideration with sonar systems is phantom targets, and has been tackled with a credibility count scheme [4].

The mapping strategy presented here is feature based. Important contributions of our research are: (i) Discrete planar and corner elements gathered by the sonar sensor at various stages are merged incrementally to form partial planes. Planar elements are only merged to the partial plane which is adjacent to them to avoid false closure, such as sealing a doorway. (ii) Our research extends 'plane to plane' and 'corner to corner' matching $[4,18]$ to exploit the relational constraint between a corner and two intersecting planes to further improve the fidelity of the map. Relational constraints are described in [1] and are used by [14] in known environments. (iii) The partial planes in our approach are used to remove some phantom targets caused by specular reflection, which would be detrimental to path planning. (iv) A new filter called here the JulierUhlmann Kalman Filter (JUKF) [20], is used to evaluate state transition equations, generate state-measurement cross covariance and propagate error covariance matrices. The JUKF method obviates the need to evaluate Jacobian matrices while improving accuracy of propagating error covariance through non-linear equations. Its performance is compared with the Iterated Extended Kalman Filter (IEKF) [6] formulated for the same purpose.

The paper is structured as follows: Section 2 describes the robot system architecture, the sonar sensor and the odometry system. Section 3 introduces the JUKF method and section 4 covers the environmental model and methods for growing the map primitives. The JUKF formulation of the mapping problem is under section 5, and section 6 discusses a method for identifying phantom targets. Map management issues of fusing new features and removal of redundancy are contained in sections 7 and 8 . This is followed by experimental results in section 9 and finally conclusions in section 10 . 


\section{System Architecture}

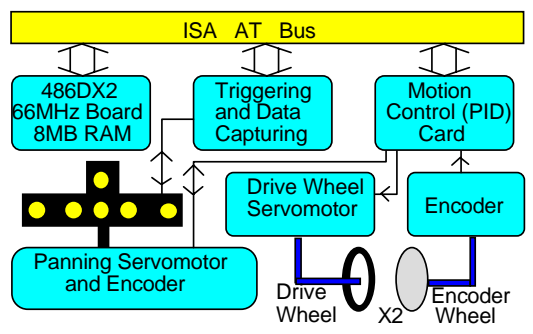

Figure 1 : The robot system architecture

The communication backbone of the robot is a ISA AT Bus. Through it a $486 \mathrm{DX} 2-66 \mathrm{MHz}$ board controls a custom made sensor control card and a motion control card. With the sensor control card, transmission of ultrasound is triggered by grounding the $300 \mathrm{~V}$-biased transducer for $10 \mu \mathrm{s}$ and the echo waveform is sampled via a 12bit $\mathrm{ADC}$ at $1 \mathrm{MHz}$. The motion control card contains a MC1401 chip which provides PID control to the four motors of pan tilt mechanism and two drive wheels. For every motor an encoder provides feedback information.

\subsection{Sonar Sensor}

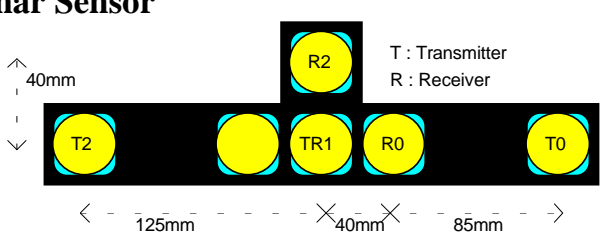

Figure 2: The sonar array configuration

The custom sonar array in Figure 2 classifies common indoor features into planes, $90^{\circ}$ corners and edges as well as accurately estimating their range and vertical and horizontal bearing angles. See [7] for a complete description. The sensor repetitively fires TR1 and listens to TR1 R0 and R2 while panning anticlockwise at $90^{\circ} / \mathrm{sec}$ to locate the directions of potential targets. Then, it pans clockwise, slowing down at the directions of the potential targets found earlier, and fires $\mathrm{T} 0$ and listens, then fires T2 and listens to classify targets.

\subsection{Odometry System}

The odometry system relies on a pair of unloaded encoder wheels made as sharp-edged as possible to reduce wheelbase uncertainty, and are independently mounted on linear bearings to allow vertical motion and minimise the problem of wheel distortion. Since the drive wheels are distinct, large accelerations can be achieved with accurate odometry. The error model used to propagate error covariance can be found in [2].

\section{JU Method of Propagating Error Covariance}

Suppose that the $n_{s} \times 1$ vector $\hat{\mathbf{S}}(k \mid k)$ is an estimate of state $\mathbf{S}(k)$ at stage $k$, and associated with the estimate is an error covariance $n_{s} \times n_{s}$ matrix $\mathbf{P}_{s s}(k \mid k)$, then by generating a set of sigma points $\sigma_{j}$ from the columns of $\pm \sqrt{n_{s} \mathbf{P}_{s s}(k \mid k)}$ A set of $2 n_{s}$ data points can be formed,

$\mathbf{S}_{i}(k \mid k) \in \bigcup_{i=0}^{n_{s}-1}\left\{\hat{\mathbf{S}}(k \mid k)+\sigma_{i}, \hat{\mathbf{S}}(k \mid k)-\sigma_{i}\right\}$

Let $\mathbf{X}(k+1)=\mathbf{X}(\mathbf{S}(k))$ and $\mathbf{Y}(k+1)=\mathbf{Y}(\mathbf{S}(k))$ be two nonlinear transformation equations in term of $\mathbf{S}(k)$. The mean of $\mathbf{X}$, the covariance of $\mathbf{X}$ and the cross covariance between $\mathbf{X}$ and $\mathbf{Y}$ can be evaluated with

$$
\begin{aligned}
& \hat{\mathbf{X}}(k+1 \mid k)=\Omega\left(\mathbf{X}(\mathbf{S}), \mathbf{P}_{s s}\right)=\frac{1}{2 n_{s}} \sum_{i=0}^{2 n_{s}-1} \mathbf{X}\left(\mathbf{S}_{i}(k \mid k)\right) \\
& \mathbf{P}_{X X}(k+1 \mid k)=\Lambda\left(\mathbf{X}(\mathbf{S}), \mathbf{P}_{s s}\right) \\
& =\frac{1}{2 n_{s}} \sum_{i=0}^{2 n_{s}-1}\left[\mathbf{X}\left(\mathbf{S}_{i}(k \mid k)\right)-\hat{\mathbf{X}}(k+1 \mid k) \llbracket \mathbf{X}\left(\mathbf{S}_{i}(k \mid k)\right)-\hat{\mathbf{X}}(k+1 \mid k)\right]^{T} \\
& \mathbf{P}_{X Y}(k+1 \mid k)=\Lambda\left(\mathbf{X}(\mathbf{S}), \mathbf{Y}(\mathbf{S}), \mathbf{P}_{s s}\right) \\
& =\frac{1}{2 n_{s}} \sum_{i=0}^{2 n_{s}-1}\left[\mathbf{X}\left(\mathbf{S}_{i}(k \mid k)\right)-\hat{\mathbf{X}}(k+1 \mid k) \llbracket \mathbf{Y}\left(\mathbf{S}_{i}(k \mid k)\right)-\hat{\mathbf{Y}}(k+1 \mid k)\right]^{T}
\end{aligned}
$$

Here $\Omega()$ and $\Lambda()$ are respectively defined as the functions for producing the mean and covariance of its input parameters. Readers are referred to [19] for the theoretical background.

\section{Environmental Model \\ 4.1 Map Primitives}

The environmental model consists of the following primitives:

Partial Plane is characterised by its state parameters $\left[\begin{array}{ll}a & b\end{array}\right]^{T}$ from the line equation $a x+b y=a^{2}+b^{2}$, the Cartesian coordinates of its approximate endpoints, and a status associated with each endpoint, indicating whether it is terminated with another partial plane to form a corner. When a wall is first detected, it is registered as a partial plane with only one endpoint. It is then grown as the robot moves along the wall.

Corner is characterised by its Cartesian coordinates $\left[\begin{array}{ll}x & y\end{array}\right]^{T}$ only. Sonar sensing cannot provide indication of orientation of corners.

In addition, the covariance and cross-covariance among these features are also kept [12]. Edge measurements are found to be generated by stray features, such as joins between wall segments, hence they are ignored at this stage. The current strategy also records the unclassifiable features as unknown. In the 
future, clusters would be formed to assist in obstacle avoidance path planning.

\subsection{Growing Map Primitives}

Since the robot is operating indoor, discrete feature elements are assumed to come from a few planes, so that they can be merged using some collinearity constraint to give a more realistic representation of the environment.

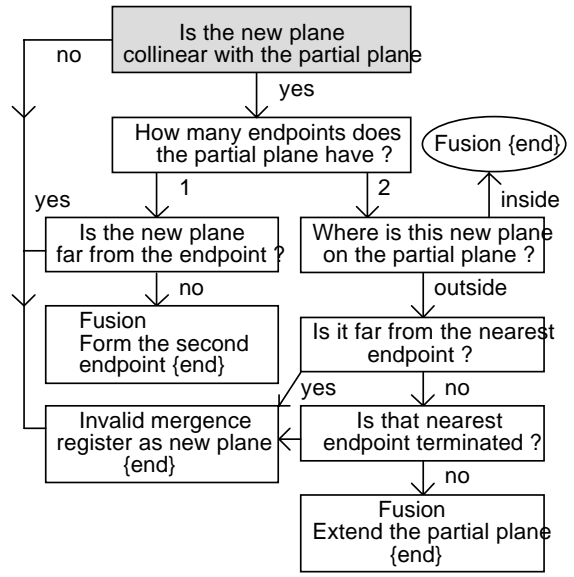

Figure 3 : Conditions for growing map primitives with a plane measurement

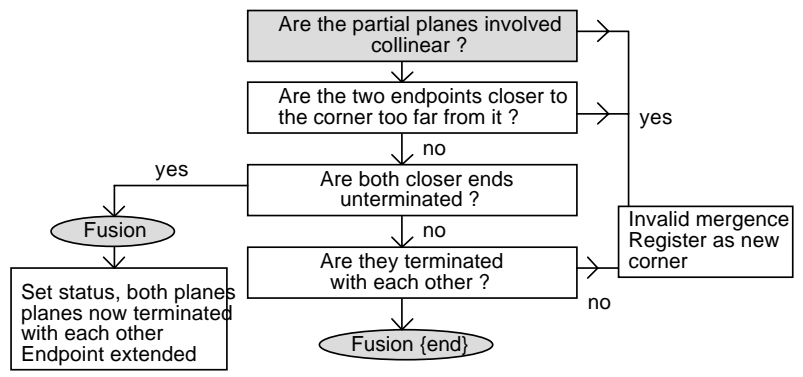

Figure 4 : Conditions for growing map primitives with a corner measurement

A planar measurement would be fused to a partial plane if it satisfies the conditions depicted in Figure 3. A corner measurement would be fused to a corner if it is close enough to it, otherwise it would be fused to two intersecting planes if it satisfies the conditions depicted in Figure 4. For all gray condition boxes in the figures, $\chi^{2}$ test (to be described later) is applied. Every time a reobservation of a feature/relation occurs, the state of every map feature would be updated because of their correlation. The unterminated endpoints of partial planes are projected to the new gradient determined by the new state parameters, whereas the terminated endpoint are re-calculated from the intersections of all pairs of partial planes marked as terminated with each other.

\section{JUKF Formulation of Mapping Problem}

A snapshot of the map building scenario at stage $k+1$ is depicted in Figure 5. The robot has just moved to a new position and sensed a few new features. It is now ready to use some features for localisation, and add the remaining features to the map.

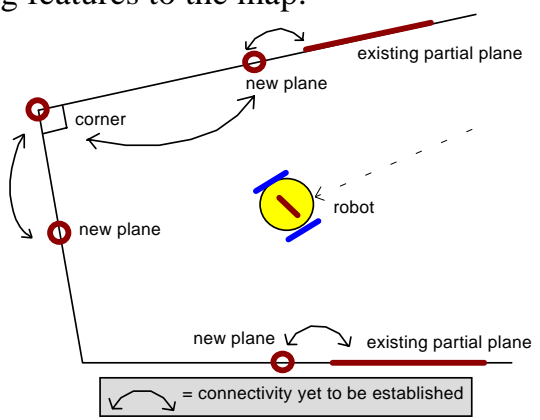

Figure 5 : Status of map and data fusion at stage $k+1$

The 2D coordinates and orientation of the robot, as well as the speed of sound at stage $k$ is denoted by the state vector $\quad \mathbf{x}_{0}(k)=\left[\begin{array}{llll}x(k) & y(k) & \theta(k) & c_{s}(k)\end{array}\right]^{T}$. Further assume that a partial map already exists, and the parameter vectors of the $N$ existing features are $\mathbf{x}_{1}(k)$, $\mathbf{x}_{2}(k), \mathbf{x}_{3}(k) \ldots$ up to $\mathbf{x}_{N}(k) . \mathbf{P}_{m n}$ is used to denoted the cross-covariance between state $m$ and state $n$.

The state transition model being used is

$\mathbf{x}_{0}(k+1)=\mathbf{F}\left(\mathbf{x}_{0}(k), \mathbf{U}(k+1)\right)$

where $\mathbf{U}(k+1)=\left[\begin{array}{ll}L(k+1) & R(k+1)\end{array}\right]^{T}$ is the amount of rotation of the left and right wheels. The new state, its covariance, and cross-covariance with all existing map features $j$ are

$\hat{\mathbf{x}}_{0}^{J U}(k+1 \mid k)=\Omega\left(\mathbf{F}\left(\hat{\mathbf{x}}_{0}, \hat{\mathbf{U}}\right), \operatorname{diag}\left(\mathbf{P}_{00}, \operatorname{Cov}(\mathbf{U})\right)\right)$

$\mathbf{P}_{00}^{J U}(k+1 \mid k)=\Lambda\left(\mathbf{F}\left(\hat{\mathbf{x}}_{0}, \hat{\mathbf{U}}\right), \mathbf{P}_{00}\right)+\operatorname{Odom}(\hat{\mathbf{U}}, \operatorname{Cov}(\mathbf{U}))$

$\mathbf{P}_{0 j}^{J U}(k+1 \mid k)=\Lambda\left(\mathbf{F}\left(\hat{\mathbf{x}}_{0}, \hat{\mathbf{U}}\right), \hat{\mathbf{x}}_{j},\left[\begin{array}{ll}\mathbf{P}_{00} & \mathbf{P}_{0 j} \\ \mathbf{P}_{j 0} & \mathbf{P}_{j j}\end{array}\right]\right)$

respectively, where $\operatorname{Odom}(.$.$) represents the new$ odometry error model developed in [2] that takes in the robot's wheel covariance matrix $\operatorname{Cov}(\mathbf{U}(k+1))$ and wheel turns $\mathbf{U}$ and outputs the propagated covariance matrix.

A measurement vector consists of a time of flight $r_{i}$ and a direction $\Psi_{i}$ to a target, and is denoted as

$$
\mathbf{M}_{i}(k+1)=\left[r_{i}(k+1) \quad \Psi_{i}(k+1)\right]^{T}
$$

Every new measurement is tested against all the possible collinearity constraints set out in section 4.2 , in order to grow the map primitives. A typical constraint would involve a new measurement $\mathbf{M}_{i}$, current position 
of the robot $\mathbf{x}_{0}$, and a few existing map features. In the following example, one existing feature $\mathbf{x}_{i}$ is involved, and the constraint equation would look like:

$$
\mathbf{G}\left(\mathbf{x}_{0}(k+1), \mathbf{x}_{i}(k+1), \mathbf{M}_{i}(k+1)\right)=\mathbf{0}
$$

Due to noise, the constraint equation does not hold exactly. This leads to the definition of residual vector (also known as innovation) required by Kalman Filter:

$$
\mathbf{z}_{i}^{J U}(k+1)=-\Omega\left(\mathbf{G}\left(\hat{\mathbf{x}}_{0}, \hat{\mathbf{x}}_{i}, \hat{\mathbf{M}}\right), \operatorname{diag}\left(\left[\begin{array}{ll}
\mathbf{P}_{00} & \mathbf{P}_{0 i} \\
\mathbf{P}_{i 0} & \mathbf{P}_{i i}
\end{array}\right], \operatorname{Cov}\left(\mathbf{M}_{i}\right)\right)\right)
$$

and its error covariance,

$$
\mathbf{P}_{z z}^{J U}(k+1 \mid k)=\Lambda\left(\mathbf{G}\left(\hat{\mathbf{x}}_{0}, \hat{\mathbf{x}}_{i}, \hat{\mathbf{M}}_{i}\right), \operatorname{diag}\left(\left[\begin{array}{ll}
\mathbf{P}_{00} & \mathbf{P}_{0 i} \\
\mathbf{P}_{i 0} & \mathbf{P}_{i i}
\end{array}\right], \operatorname{Cov}\left(\mathbf{M}_{i}\right)\right)\right)
$$

Since all the measurement vectors are assumed uncorrelated, block processing is not required. Also, if a corner measurement is found to lie on one partial plane only, fusion is not carried out because only a $1 \times 1$ collinearity residual vector can be formed with the $2 \times 1$ corner measurement. Discarding the corner measurement after use wastes some useful information, but registering the corner as a new primitive after fusion introduces redundancy which results in a near singular global error covariance matrix. More complex map management schemes, such as reparameterisation, would then become necessary.

The constraint is only considered valid if it satisfies a $95 \%$ confidence $\chi^{2}$ test:

$\left\|\mathbf{z}_{i}\right\|_{\mathbf{P}_{z z}}^{2}=\mathbf{z}_{i}^{T}(k+1) \mathbf{P}_{z z}^{-1}(k+1 \mid k) \mathbf{z}_{i}(k+1)<\chi_{95 \%}^{2}$

To update the state of every existing feature $\mathbf{x}_{j}$, the cross-covariance between $\mathbf{x}_{j}$ and $\mathbf{z}_{i}$ is required.

$\mathbf{P}_{j z}^{J U}(k+1 \mid k)=\Lambda\left(\hat{\mathbf{x}}_{j}, \mathbf{G}\left(\hat{\mathbf{x}}_{0}, \hat{\mathbf{x}}_{i}, \hat{\mathbf{M}}_{i}\right), \operatorname{diag}\left(\left[\begin{array}{ccc}\mathbf{P}_{00} & \mathbf{P}_{0 i} & \mathbf{P}_{0 j} \\ \mathbf{P}_{i 0} & \mathbf{P}_{i i} & \mathbf{P}_{i j} \\ \mathbf{P}_{j 0} & \mathbf{P}_{j i} & \mathbf{P}_{j j}\end{array}\right], \operatorname{Cov}\left(\mathbf{M}_{i}\right)\right)\right)$

then applying Kalman update equations [14],

$$
\hat{\mathbf{x}}_{j}(k+1 \mid k+1)=\hat{\mathbf{x}}_{j}(k+1 \mid k)+\mathbf{P}_{j z}(k+1 \mid k)
$$

$$
\mathbf{P}_{z z}^{-1}(k+1 \mid k) \mathbf{z}_{i}(k+1)
$$

and for all combinations of existing features $\mathbf{x}_{m}$ and $\mathbf{x}_{n}$,

$$
\begin{gathered}
\mathbf{P}_{m n}(k+1 \mid k+1)=\mathbf{P}_{m n}(k+1 \mid k)-\mathbf{P}_{m z}(k+1 \mid k) \\
\mathbf{P}_{z z}^{-1}(k+1 \mid k) \mathbf{P}_{z n}(k+1 \mid k)
\end{gathered}
$$

The process is then repeated until all observations have been processed. The formulation involving two states is similar. The actual implementation also adopts the principle of Relocation-
Fusion introduced by [12] which lessens the sensitivity to position bias at the expense of optimality. The equation development is similar to [12] and are not shown here. The remaining features are considered new and are simply incorporated into the global state vector, following its estimation. More information is contained in section 7 .

\section{Distinguishing Phantom Targets}

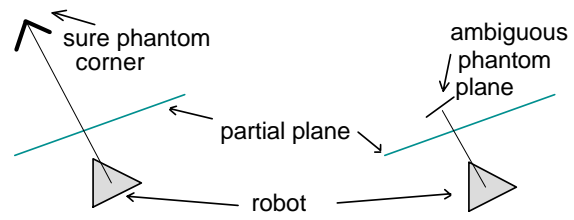

Figure 6 : Example of treatment of phantom targets

Robot positions from which features are observed are stored. When the map is sufficiently complete, many phantom targets caused by specular reflection can be eliminated by checking whether the line of sights from the positions they were observed are blocked by some partial planes. If the phantom targets are too close to some partial planes they are considered ambiguous and would not be eliminated.

\section{Fusion of Remaining New Features}

After localisation, the fusion of the remaining features will make use of the estimated robot position. For each new feature $\mathbf{x}_{i}$

$\mathbf{x}_{i}(k+1)=\mathbf{H}\left(\mathbf{x}_{0}(k+1), \mathbf{M}_{i}(k+1)\right)$

hence

$\hat{\mathbf{x}}_{i}^{J U}(k+1 \mid k+1)=\Omega\left(\mathbf{H}\left(\hat{\mathbf{x}}_{0}, \hat{\mathbf{M}}_{i}\right), \operatorname{diag}\left(\mathbf{P}_{00}, \operatorname{Cov}\left(\mathbf{M}_{i}\right)\right)\right)(18$

$\mathbf{P}_{i i}^{J U}(k+1 \mid k+1)=\Lambda\left(\mathbf{H}\left(\hat{\mathbf{x}}_{0}, \hat{\mathbf{M}}_{i}\right), \operatorname{diag}\left(\mathbf{P}_{00}, \operatorname{Cov}\left(\mathbf{M}_{i}\right)\right)\right)$

and all the cross-covariance among the new and the existing features are also generated. Let $\mathbf{x}_{j}, \forall j \neq i$ denote the objects already in the map,

$\mathbf{P}_{i j}^{J U}(k+1 \mid k+1)=\Lambda\left(\mathbf{H}\left(\hat{\mathbf{x}}_{0}, \hat{\mathbf{M}}_{i}\right), \hat{\mathbf{x}}_{j}, \operatorname{diag}\left(\left[\begin{array}{ll}\mathbf{P}_{00} & \mathbf{P}_{0 j} \\ \mathbf{P}_{j 0} & \mathbf{P}_{j j}\end{array}\right], \operatorname{Cov}\left(\mathbf{M}_{i}\right)\right)\right)$

\section{Removal of Redundancy}

This occurs when (i) two existing partial planes are actually collinear and adjacent to each other, (ii) two existing corners are the same (iii) an existing corner appears to be located at the intersection of two existing partial planes. In such cases, internal fusion is performed by forming residual vectors in a manner similar to section 5 (without the measurement vector), for example, in case (iii) three states are involved,

$\mathbf{G}\left(\mathbf{x}_{i}, \mathbf{x}_{j}, \mathbf{x}_{k}\right)=\mathbf{0}$ 
If internal fusion is successful, in the first case, one of the partial planes is discarded (together with its covariance and cross covariance with other features) and the endpoints of the other are updated; In the second case, one of the corner is discarded; In the third case, the corner is discarded and the endpoints of the two partial planes are updated.

\section{Experimental Results}

Experiments have been carried out in four structured environments erected with cardboard boxes. Only one set of results are shown here. The odometry of the robot has been calibrated to reduce systematic errors, and the parameters required by the non-systematic error model have been obtained in [2] prior to experiment.

Since the boxes were lined up manually using the parquetry flooring, the variance associated with the time of flight measurement and angular measurement were set larger than that achievable by the sonar sensor [7] in order for the collinearity constraints to hold. For all experiments, the following values were used:

- standard deviation of time of flight $=16 \mu \mathrm{s}$

- standard deviation of direction $=3.5^{\circ}$

- initial value of speed of sound, $c_{s}=342.5 \mathrm{~m} / \mathrm{s}$

- initial standard deviation of $\quad c_{s}=0.18 \mathrm{~m} / \mathrm{s}$

The first two standard deviations could be significantly reduced for real walls rather than boxes. The results are shown in Figure 7(a)-(d) with a 1 metre grid. 7(a) shows the actual environment. 7(b) shows all the sonar features detected at various positions (before position correction) being superimposed on the same diagrams, and the 'scan lines' from one of the position indicate the typical number of features the sonar sensor can capture at any one time. The sensor detects the gaps between the boxes as edges. 7(c) and 7(d) are the IEKF and JUKF generated maps respectively.

In the experiment, Werrimbi was programmed to repetitively enter, make a $180^{\circ}$ turn, leave an enclosure four times to investigate the long term performance of both filters. Both filters had remained consistent until the final 'exit' from the enclosure when two partial planes are formed where one plane is physically present. Nevertheless, other partial planes are consistent and all standalone corner features have been fused to form intersecting partial planes. Various phantom corners (and the clusters of unknown around them) can be observed, and the corners at $(0.2 \mathrm{~m},-1 \mathrm{~m})$ and $(2.5 \mathrm{~m}$, $1.5 \mathrm{~m}$ ) (the top left is the origin) have been eliminated because they are blocked by a partial plane. The corner at $(2.3 \mathrm{~m},-2.4 \mathrm{~m})$ has been considered ambiguous but the one at $(5 \mathrm{~m},-1 \mathrm{~m})$ has been considered valid because it is not blocked by any plane.

\section{Conclusion and Future Work}

The capability of autonomous navigation by mapping of our mobile robot system in a simple environment has been demonstrated. JUKF has proven useful and also comparable to IEKF. The mapping algorithm is now being upgraded to enhance its robustness. Current research focal points include the incorporation of edge measurement into primitive growth, clustering of unknown targets for the purpose of path planning, and also a map matching strategy to re-establish robot's position when its uncertainty is too large.

\section{Acknowledgment}

Mr. Greg Curmi's assistance in designing the robot is gratefully acknowledged.

\section{Reference}

[1] Ayache, N. and Faugeras, O.D. "Maintaining Representation of the Environment of a Mobile Robot", IEEE Transactions on Robotics and Automation, Vol 5, No 6, Dec 1989, pp.804-819.

[2] Chong, K.S. and Kleeman, L. "Accurate Odometry and Error Modelling for a Mobile Robot", 1997 International Conference on Robotics and Automation (this proceedings)

[3] Dudek, G. et al "Just-in-time Sensing: Efficiently Combining Sonar and Laser Range Data for Exploring Unknown Worlds", Proceedings 1996 IEEE International Conference on Robotics and Automation, pp.667-672.

[4] Durrant-Whyte, H.F. and Leonard, J.J. "Simultaneous Map Building and Localisation for an Autonomous Robot", IEEE/RSJ International Workshop on Intelligent Robots and Systems IROS '91, Nov 3-5, 1991, pp.14421447.

[5] Gonzalez, J. "An Iconic Position Estimator for a 2D Laser RangeFinder" Proceedings 1992 IEEE International Conference on Robotics and Automation, Vol 3, pp.26462651.

[6] Jazwinski, A.H. "Stochastic Processes and Filtering Theory", New York: Academic Press, 1970.

[7] Kleeman, L. and Kuc, R. "Mobile Robot Sonar for Target Localization and Classification", The International Journal of Robotics Research, Vol. 14, No 4, August 1995, pp.295-318.

[8] Kuc, R. and Siegel, M.W. "Physically Based Simulation Model for Acoustic Sensor Robot Navigation", IEEE Transactions on Pattern Analysis and Machine Intelligence, Vol PAMI-9, No 6, Nov 1987, pp.766-778.

[9] Leonard, J.J. and Durrant-Whyte H.F. "Dynamic Map Building for an Autonomous Mobile Robot", International Journal of Robotics Research, August 1992, Vol 11, pp.286-298.

[10] Lim, J.H. and Cho, D.W. "Experimental Investigation Of Mapping and Navigation Based on Certainty Grid Using Sonar Sensors", Robotica, Jan-Feb 1993, Vol 11, Iss:part 1, pp.7-17. 
[11] Lu, F. and Milios, E.E. "Optimal Global Pose Estimation for Consistent Sensor Data Registration", Proceedings 1995 IEEE International Conference on Robotics and Automation, pp.93-100.

[12] Moutarlier, P. and Chatila, R. "Stochastic Multisensory Data Fusion for Mobile Robot Location and Environment Modeling", 5th International Symposium on Robotics Research, Tokyo, 1989, pp.85-94.

[13] Moravec, H.P. and Elfes, A. "High Resolution Map From Wide-Angle Sonar", Proceedings 1985, IEEE International Conference on Robotics and Automation, pp.116-121.

[14] Neira, J. et al "Multisensor Mobile Robot Localisation" Proceedings 1996 International Conference on Robotics and Automation, pp.673-679.

[15] Ohya, A. et al "Exploring Unknown Environment and Map Construction Using Ultrasonic Sensing of Normal Direction of Walls", IEEE International Conference on Robotics and Automation, 1994, pp.485-492.

(a) Environment

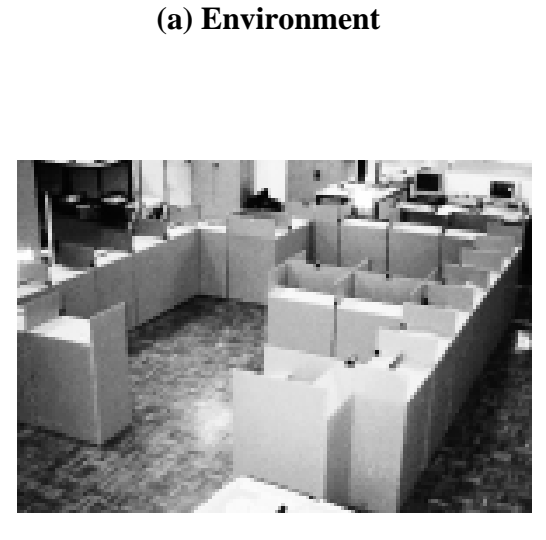

(c) Post-IEKF

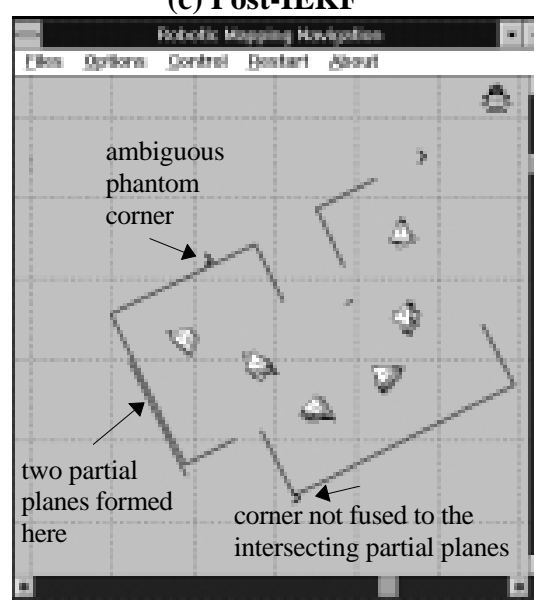

[16] Oriolo, G. et al "On-Line Map Building and Navigation for Autonomous Mobile Robots", IEEE International Conference on Robotics and Automation, 1995, pp.29002906.

[17] Pagac, D. et al "An Evidential Approach to Probabilistic Map-Building”, Proceedings 1996 IEEE International Conference on Robotics and Automation, pp. 745-750.

[18] Rencken, W.D. "Concurrent Localisation and Map Building for Mobile Robots Using Ultrasonic Sensors", Proceedings 1993 IEEE International Conference On Robotics and Automation, Vol 3, pp.2192-2197.

[19] Smith, R.C. and Cheeseman, P. "On the Representation and Estimation of Spatial Uncertainty", The International Journal of Robotics Research, Vol 5, No 4, 1986, pp.5668.

[20] Julier, S. and Uhlmann, J. Durrant-Whyte, H. "A New Approach for Filtering Nolinear Systems", Proc 1995 American Control Conference, Seattle, Washington, USA.
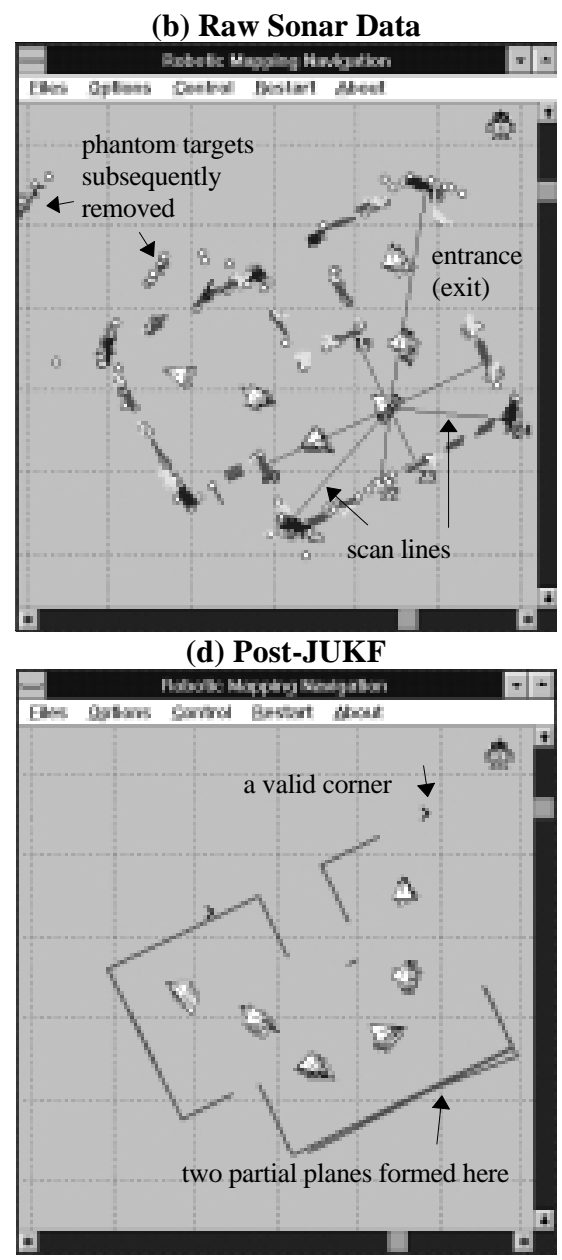

Figure 7 : Comparison of (a) actual environment, (b) raw sonar data before filtering, (c) map generated by JUKF and (d) map generated by IEKF, for a structured environment. In the pre-filtering map, short line segment indicates plane, light colored pointy symbol is edge, dark colored pointy symbol is corner, circle is unknown and finally the white triangle is the robot. 